\title{
Quantitative microbial risk assessment modelling for the use of reclaimed water in irrigated horticulture
}

\author{
A. J. Hamilton ${ }^{1}$, F. Stagnitti ${ }^{1}$, A.-M. Boland ${ }^{2}$ \& R. Premier ${ }^{2}$ \\ ${ }^{1}$ School of Ecology and Environment, Deakin University, Australia \\ ${ }^{2}$ Department of Primary Industries Victoria, Australia
}

\begin{abstract}
Reuse of treated sewage effluent for the irrigation of horticultural crops is being propounded and practiced as a means of alleviating pressure on freshwater resources. Concerns have been raised, however, as to the risk to human health, primarily disease, associated with this practice. Quantitative Microbial Risk Assessment (QMRA) is a useful tool for estimating this risk. We describe how QMRA works and the current state of knowledge of the components of QMRA models for the horticultural reuse scenario.

Keywords: food-safety, horticulture, pathogen, quantitative microbial risk assessment, reclaimed water, recycled water, vegetable.
\end{abstract}

\section{Introduction}

Increasing human population sizes are placing significant strain on the World's freshwater resources. Rivers are becoming polluted, extraction for agricultural use is substantially reducing flows, and competition for freshwater is intensifying. Moreover, with the growth of large cities, there is a concomitant escalation in sewage output, and thus a likely increase in detrimental effects associated with discharge of effluent to receiving waters. One means of alleviating such stresses is to reuse wastewater for horticultural irrigation (Hamilton et al. [1]). But this practice has been approached with a degree of trepidation, owing primarily to concerns about risks to human health via contamination of food with pathogenic microorganisms [1]. In theory at least, such risks can be mitigated through combinations of low- and high-technology engineering solutions. At the recent Integrated Concepts in Recycled Water 
conference (Wollongong, February 2005), Professor Don Bursill made this pertinent observation: "You can turn anything wet into drinking water if you filter it through enough money".

But this is also where risks can enter a system: it is usually not economically feasible to employ almost fail-safe treatment. Little wastewater in the developing world undergoes treatment of any kind, and even in affluent countries the cost of treatment is a key criterion determining the likely success or failure of a reuse scheme (Robinson [2]). Thus, we need methods to determine the risk that different recycled water irrigation scenarios pose to human health, so that safe and economically realistic schemes can be developed.

Recent years have seen a general movement towards the use of QMRA for determining health risks. The brief for the revised World Health Organisation (WHO) guidelines for agricultural reuse (due for release in 2005) was to use QMRA (Blumenthal et al. [3]). Australia is also currently revising its national guidelines for recycled water use, and these will include QMRA (Cunliffe et al. [4]). The purpose of this review is to outline how QMRA works, and to progress ideas on how it can best be applied to horticultural reuse scenarios. Knowledge gaps relating to such QMRA models are identified and subsequent recommendations for strategic research are made.

\section{What is Quantitative Microbial Risk Assessment (QMRA)?}

Quantitative Risk Assessment (QRA) modelling is a formal process for calculating probabilities of risk associated with defined scenarios. It was originally developed to determine risks to humans of environmental exposure to various hazards, especially chemicals (NRC [5]). Recent times have seen the emergence of a specific form of QRA, namely, QMRA. QMRA comprises four distinct steps: (i) hazard identification, (ii) exposure assessment, (iii) doseresponse analysis, and (iv) risk characterisation (Haas et al. [6]).

The first QMRAs for reclaimed water irrigation of food crops were simplistic models where the various parameters - such as the concentration of viruses in the irrigation water or the amount of food consumed - were represented as pointestimates (Asano and Sakaji [7], Asano et al. [8], Rose et al. [9], Shuval et al. [10]). More recent models have used probability distributions to define parameters (Tanaka et al. [11], van Ginneken and Oron [12], Petterson et al. [13]). This is an important step forward, as it means that a more accurate estimate of risk can be calculated, since variability and uncertainty are accounted for. QMRA is applied to a conceptual model such as the one we present below for the irrigation of horticultural crops with treated wastewater, fig. 1.

\section{Hazard identification}

In the context of QMRA, hazard identification primarily involves determining the microbiological agent(s) likely to be of potential significance with respect to human health. There have been many reported cases of human pathogen 


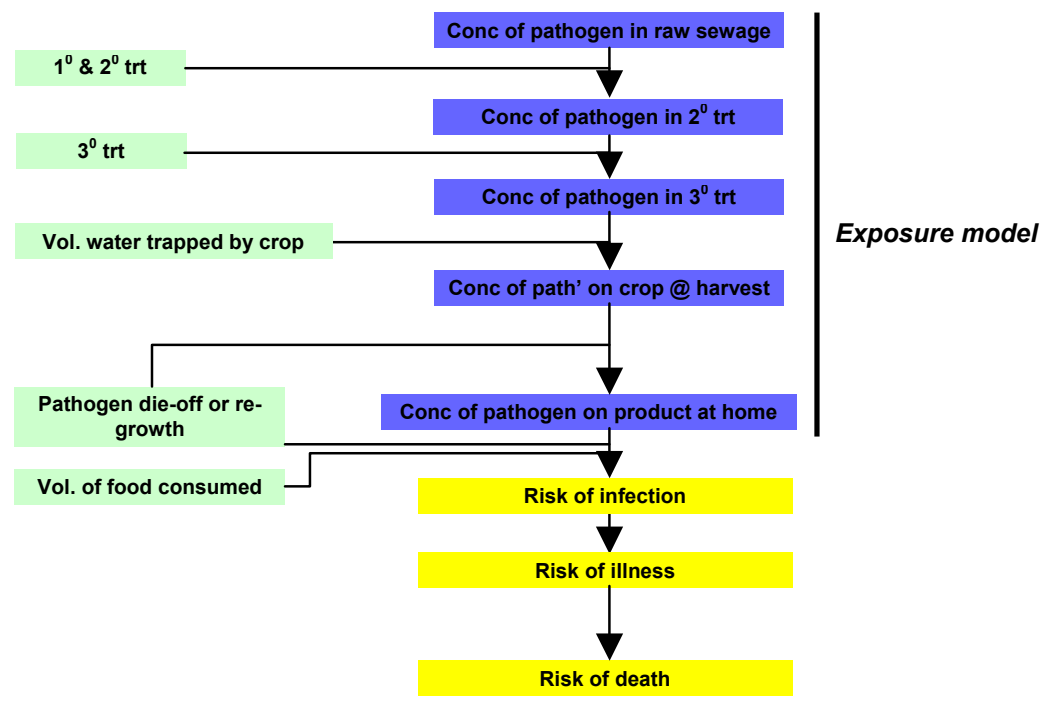

Figure 1: Conceptual model of risk pathway associated with consumption of horticultural crops irrigated with wastewater.

contamination of crops irrigated with reclaimed water (summarised by Fegen et al. [14]. But establishing a cause-effect relationship between contaminated produce and disease outbreaks has proved rather difficult. One of the more convincing cases is the 1970 cholera outbreak in Jerusalem (Shuval et al. [15], Fattal et al. [16]). Illicit irrigation of vegetables with reclaimed water and subsequent purchase by around 100,000-200,000 people was followed by about 200 cases of cholera. The cause-effect connection was strengthened by the fact that the risk analysis model of Shuval et al. [10], when applied to this irrigation scenario, proved to be a good predictor of the magnitude of the epidemic. There is also reasonable evidence to suggest that outbreaks of cholera and typhoid fever in Chile in the late 1970 s to early 1980 s could be attributed to contaminated food from wastewater-irrigated horticulture (Shuval [17]).

Recent QMRA models have primarily been concerned with risk of disease from enteric viruses $[11,12,13]$. The rationales for using viruses are that they (i) are generally highly persistent in the environment, (ii) have low-dose infectivity, and (iii) are relatively difficult to remove/inactivate in wastewater treatment systems. Asano et al. [8] have argued that the difficulty of routine monitoring for enteric viruses is another reason for developing QMRAs that address this group. A wide variety of enteric viruses are found in wastewaters (Irving [18]). The most commonly problematic from a human health perspective are enteroviruses, rotaviruses, hepatitis A virus, and Norwalk or Norwalk-like viruses. 


\section{Exposure assessment}

The objective of exposure assessment is to determine the quantity of pathogenic organisms consumed by a person eating or drinking the product of interest. This can be achieved relatively easily for drinking water scenarios by determining the concentration of pathogens in the water and the amount of water typically consumed by an individual (Haas et al. [19]). But the situation is somewhat more complex when considering horticultural irrigation. In addition to information on the concentration of pathogens in the raw wastewater and the amount of food consumed, factors such as treatment efficiency, irrigation method, and pathogen die-off need to be accounted for.

\subsection{Pathogen concentration in wastewater}

Asano et al. [8] collated the largest database in the USA for enteric virus concentrations in treated wastewater. It comprises 424 secondary-treated effluent samples and 814 tertiary-treated samples. The concentration of pathogens such as enteric viruses in tertiary-treated effluent is a function of the concentration in the secondary-effluent and the efficiency of the tertiarytreatment process. Possibly the most comprehensive study of a tertiary-treatment plant's pathogen removal efficiency is that of Rose et al. [9], table 1. Parkinson and Roddick [20] reviewed the literature on the pathogen removal efficiencies of fourteen different wastewater treatment technologies, and categorised them into broad efficiency classes.

Table 1: Pathogen removal efficiencies (as \%, with log removals in parentheses) of different tertiary treatment processes. After Rose et al. [9].

\begin{tabular}{llllll}
\hline Pathogen & $\begin{array}{l}\text { Biological/ } \\
\text { clarification }\end{array}$ & Filtration & Chlorination & $\begin{array}{l}\text { Storage } \\
(16-24 \mathrm{hr})\end{array}$ & $\begin{array}{l}\text { Complete } \\
\text { treatment }\end{array}$ \\
\hline Enteroviruses & 98.0 & 84.0 & 96.5 & 90.91 & 99.999 \\
& $(1.71)$ & $(1.71)$ & $(1.45)$ & $(1.04)$ & $(5.01)$ \\
Giardia & 93.0 & 99.0 & 78.0 & 49.5 & 99.993 \\
& $(1.19)$ & $(2.00)$ & $(0.65)$ & $(0.30)$ & $(4.14)$ \\
Cryptosporidium & 92.8 & 97.9 & 61.1 & 8.5 & 99.95 \\
& $(1.14)$ & $(1.68)$ & $(0.41)$ & $(0.04)$ & $(3.27)$ \\
Helminths & $>75.0$ & no data & no data & no data & $<99.6$ \\
\hline
\end{tabular}

\subsection{Volume of water on crop and attachment of pathogens to crop}

A critical step in the development of horticultural reuse QMRAs is the determination of the volume of water caught by plants. The concentration of pathogens on the plant at harvest is plainly a function of this. Surprisingly, there is a dearth of information on the process. Glass-house experiments simulating spray-irrigation of cucumber and lettuce revealed that on average 0.36 and 10.8 $\mathrm{mL}$ of water was retained by $100 \mathrm{~g}$ of each product, respectively [10]. The latter point-estimate was subsequently used by Petterson et al. [13] in their lettuce 
irrigation QMRA. Aside from the theoretical limitations associated with point estimates, it should be noted that Shuval et al.'s estimates are based on small sample sizes: 26 and 12 for cucumber and lettuce, respectively. Moreover, it appears that each vegetable was represented by one cultivar only (the cultivars were not reported, but the lettuce was referred to as 'long leaf'). While cultivar effects are likely to be trivial for cucumbers, they may be significant for more structurally complex and diverse plants like lettuce. In an unpublished fieldstudy we irrigated three cultivars of lettuce-Iceberg, Cos, and Romaine - and measured the amount of water trapped by 25 plants of each. Analysis of variance on $\log _{10}$-transformed data demonstrated a significant cultivar effect $(P=$ $\left.0.045, d f_{\text {total }}=74\right)$, and post hoc Least Significant Difference tests revealed a significant difference $(P<0.05)$ between Iceberg $(37.3 \mathrm{~mL})$ and Romaine $(26.8$ $\mathrm{mL})$, with neither of these cultivars being significantly different from Cos (30.7 $\mathrm{mL}$ ). Data on mass were not recorded, so it is not possible to compare these values to those of Shuval et al. [10]. Nonetheless, it does highlight the potential significance of cultivar variation.

\subsection{Pathogen decay}

Pathogens on a plant's surface, being exposed to sunlight, are susceptible to desiccation and inactivation or death by ultra-violet light. A significant positive correlation between mean hours of monthly sunshine and the rate of die-off has been demonstrated for bacteria on salad crops (Fattal et al. [21]). Schwartbrod [22] estimated that there would be $a<6 \log _{10}$ reduction in virus concentration on a plant from the time of irrigation to consumption. While useful, this is a very rough estimate, and QMRAs would probably be best to use models that describe decay rates as a function of time (and possibly other factors). Moreover, it may be fruitful to break down the production and distribution chain into field and post-harvest events when addressing pathogen decay.

The decay of pathogens in the environment can be modelled using a simple first-order rate equation such as

$$
f=\mu_{1} / \mu_{0}=e^{(-k t)}
$$

where $f$ is the proportion of pathogens remaining (viable) after time $t(\mathrm{~d}), \mu_{0}$ and $\mu_{1}$ are the respective initial and final pathogen concentrations, and the slope parameter $k$ is the decay coefficient $\left(\mathrm{d}^{-1}\right)$. Asano and Sakaji [7] and Asano et al. [8] used this function in their deterministic models. They were concerned with a variety of risks (vegetable and golf-course irrigation, recreational waters, and groundwater recharge) and assumed a generic $k$ for enteric viruses of $0.69 \mathrm{~d}^{-1}$; a justification for this value was not given. van Ginneken and Oron [12] subsequently used this estimated $k$-value in their stochastic model. Experiments on the decay of Bacteriodes fragilis bacteriophage B40-8 on glasshouse-grown lettuce yielded an estimated $k$ of $0.47 \mathrm{~d}^{-1}$ (Petterson et al. [23]).

In the log-linear model in eqn. (1) the probability of a pathogen being inactivated remains constant over time and is described by the parameter $k$. 
Thus, inactivation is assumed to be a single-phase process, and the model does not accommodate multi-phase inactivation kinetics that may be appropriate where persistent sub-populations exist. Petterson et al. [23] fitted the following bi-phasic inactivation model to their bacteriophage survival data on lettuce and carrot crops:

$$
\mu_{1}=\mu_{0}\left[a e^{-k_{1} t}+(1-a) e^{-k_{2} t}\right\rfloor
$$

where $a$ is a mixing parameter that describes the proportionate size of the subpopulation, $k_{1}$ and $k_{2}$ are the respective decay coefficients for the main and subpopulations, and $t, \mu_{0}$, and $\mu_{1}$ are as defined above. For both crops the bi-phasic model showed a statistically superior fit over the single-phase, thus inferring the presence of persistent sub-populations.

The $k$ parameter(s) in eqn. (1) and eqn. (2) are not the only constants that require careful consideration when developing a QMRA model. The time, $t$, that the viruses are exposed to the environment could have a marked effect on the model output. Asano et al. [8] assumed that irrigation would cease two weeks before harvest, and they consequently assigned a $t$-value of $14 \mathrm{~d}$ to their model. In doing so they implicitly assumed that no virus inactivation took place after harvest. Petterson et al. [13] also used $t=14 \mathrm{~d}$, but stated that consumption was assumed to take place this long after the last irrigation event. van Ginneken and Oron [12] adopted a novel approach whereby the elapsed time between final irrigation and consumption was optimised for their specific QMRA so as to obtain a total risk of infection of $<10^{-4}$ (i.e. to comply with US EPA [24]). The optimal value was $20 \mathrm{~d}$ for spray irrigation of vegetables.

\subsection{Pathogen re-growth}

Viruses are not able to grow outside their hosts. Thus, considering most QMRA models for horticultural re-use have been based on viruses, pathogen re-growth has not needed to be addressed. Bacterial and fungal pathogens, on the other hand, do have the potential to re-grow on the produce. Several models that describe growth as function of time and environmental variables have been constructed (see review by López et al. [25]). Detailed re-growth models for pathogens on vegetables are generally lacking.

\subsection{Quantity of food consumed}

Quantification of the amount of food consumed is an important step in a QMRA model. Unfortunately, unlike drinking water scenarios, Rosebury and Burmaster [26], comprehensive data on consumption rates are difficult to obtain. In their model, Shuval et al. [10] simply used point-estimates, and assumed that Israelites consumed $100 \mathrm{~g}$ of lettuce or cucumber $\mathrm{d}^{-1}$ for $150 \mathrm{~d}$. Petterson et al. [13] based their assumption of $100 \mathrm{~g}$ of lettuce per consumption event on Shuval et al.'s point-estimate. Asano et al. [8] and Tanaka et al. [11] assumed that consumers of crops irrigated with reclaimed water were exposed to an average 
daily dose of $10 \mathrm{~mL}$ of reclaimed water. van Ginneken and Oron [12] used a more realistic approach, whereby consumption behaviour of raw fruits and vegetables (combined) was described using probability distributions.

\section{Dose-response models}

Dose-response models describe the relationship between the mean number of microorganisms ingested and the probability of a specific outcome (usually infection) in individuals that have received that mean dose (Vose [27]). The two most commonly-used dose-response models in QMRA are the exponential and the $\beta$-Poisson. The best-fit parameters for the models are determined from trials where subjects are challenged with specific doses of a pathogen or a substitute for it.

The exponential model is:

$$
P_{\text {inf }}(\lambda ; r)=1-e^{(-r \lambda)},
$$

where $\lambda$ is the number of potentially infective microorganisms consumed and $r$ is the probability that each microbe has of causing infection to a consumer (pathogen-host survival probability). The model carries the following assumptions: (i) there is a constant probability of infection across all individuals in a population, (ii) each individual microorganism has the same infective capability, and (iii) survival of a microorganism within a host is independent of the survival of any other microorganism in the same host. The exponential model has found particular application for enteric protozoan pathogens such as Cryptosporidium parvum and Giardia lamblia, which are typically found in low numbers [6].

The $\beta$-Poisson dose-response model, derived by Furumoto and Mickey [28], is an extension of the exponential. In addition to describing $\lambda$ with a probability function (the Poisson), $r$ is described with the $\beta$-probability distribution. Thus, the $\beta$-Poisson infectivity model is

$$
P_{\text {inf }}(\lambda ; \alpha, \beta)=1-{ }_{1} F_{1}(\alpha, \alpha+\beta,-\lambda)
$$

where $\alpha$ and $\beta$ are fit-parameters (but also have biological meaning, [27], and ${ }_{1} F_{1}()$ is the Kummer confluent hypergeometric function. This model cannot readily be fitted to dose-response data to obtain maximum likelihood estimates of its parameters. The problem is circumvented by using an approximation, [28]. If $\beta>>1$ and $\beta>>\alpha$ then $P_{\text {inf }}$ can be estimated as

$$
P_{\mathrm{inf}}=1-(1+\lambda / \beta)^{-\alpha}
$$

This approximation of the $\beta$-Poisson has been used widely in QMRA. 
The $\beta$-Poisson is a useful alternative to the exponential as it allows one to incorporate individual variability, both in the pathogen and host. This property is particularly valuable where subpopulations, such as pregnant women, immunodeficient people (e.g. AIDS sufferers), children, or the elderly, are prevalent or of particular interest. Thus, the $\beta$-Poisson is generally more appropriate than the exponential for pathogens that illicit an immune response. A $\beta$-Poisson dose-response model for rotavirus infection of humans was developed by Ward et al. [29], and has this been widely used for drinking water, $[9,11]$, and horticultural reuse QMRAs $[12,13]$. Rotaviruses form a sub-group of enteric viruses. They are known for being highly infective, and are particularly common in susceptible sub-populations such as the young and the elderly. They are thus considered to represent an adequate worst-case scenario of enteric viruses. Best-fit parameters of other dose response studies were tabulated by Hass et al. [6].

\subsection{Risk characterisation}

Risk characterisation combines the exposure and dose-response models so that a risk probability can be estimated. As alluded to above, risk can be determined in one of either two ways. Point estimates of model parameters can be used and a single probability describing risk calculated. Alternatively, the model parameters can be represented by probability distributions, which can be sampled from at random many times over using computer simulation techniques such as Monte Carlo. Such stochastic models are preferred because they accommodate variation. More specifically, they can account for variability and uncertainty. Variability is the natural variation among data. It cannot be reduced through improved experimental design or measurement techniques. Uncertainty, on the other hand, represents one's ignorance about the parameter of interest. It can be reduced through more accurate and/or precise sampling. It is not always possible to separate variability and uncertainty, but simply including variation, or total uncertainty (Vose [30]), in a QMRA model gives a truer representation of risk than simple point-estimate models $[6,30]$.

The discussion hitherto has been concerned with determining the level of risk. An equally important agendum is defining the acceptable level of risk. There has been little guidance for setting suitable risk levels for reuse scenarios. To this end, QMRA models for effluent reuse have generally followed the lead of the drinking water industry. The US EPA [24] set an arbitrary acceptable risk level of one infection per 10,000 people per year (i.e. $<10^{-4}$ ) for human consumption of drinking water. This has been used as the critical risk level or reference point for several wastewater reuse QMRAs [8, 9, 10, 11 15]. The appropriateness of this standard was questioned by Haas [31] who propounded a more conservative benchmark of $10^{-3}$.

Recent years have seen the emergence of a new approach to setting acceptable risk standards - the disability adjusted life year (DALY) (WHO [32]). The DALY metric is calculated as the sum of years of life lost (YLL) due to 
premature death and the number of years lived with a disability (standardised for severity) (YLD). Thus,

$$
\mathrm{DALY}=\mathrm{YLL}+\mathrm{YLD}
$$

The exact calculation of YLL and YLD is complex and is outlined by WHO [32]. The DALY is the chosen metric of acceptable risk for the WHO's new drinking water guidelines WHO [33] and their revised recycled water guidelines that are in progress (Carr et al. [34]). It is also to be used in the revised Australian recycled water guidelines.

\subsection{Conclusion}

The use of reclaimed water in irrigated horticulture is a contentious issue, owing primarily to concerns about the microbiological safety of produce grown in such a manner. QMRA has much to offer the debate. It will allow objective characterisation of risks associated with different scenarios. It is already starting to have an impact as several key reuse guidelines are based on it. Nonetheless, there is still substantial scope for improved QMRA models. Data upon which the models are based are still poor for certain steps, especially (i) the volume of water trapped by a plant, (ii) pathogen decay kinetics, (iii) and produce consumption behaviour of consumers. Also, most models are restricted to one or a few scenarios. Computerised decision support tools wherein a schemedeveloper could run a QMRA for a specifically-defined scenario could be developed in the future, and could prove useful for viability and safety of the scheme.

\section{References}

[1] Hamilton, A. J., Boland, A.-M., Stevens, D., Kelly, J., Radcliffe, J., Ziehrl, A., Dillon, P. J. \& Paulin, R., Position of the Australian horticultural industry with respect to the use of reclaimed water. Agricultural Water Management, 71, pp. 181-209, 2005.

[2] Robinson, J., Cost benefit analysis of effluent re-use schemes: why water recycling projects fail to pass the test. Water recycling Australia: second national conference, Brisbane, 2003. CD ROM.

[3] Blumenthal, U. J., Peasey, A., Ruiz-Palacios, G. \& Mara, D., Guidelines for Wastewater Reuse in Agriculture and Aquaculture: Recommended Revisions Based on New Research Evidence. WELL Study, Task No. 68, Part 1. Water and Environmental Health at London and Loughborough (WELL), London, UK, 2000.

http:/www.lboro.ac.uk/well/resources/well-studies/well-studies.htm.

[4] Cunliffe, D. A., Bursill, D. \& Hooy, T., Developing national guidelines on water recycling. Integrated Concepts in Water Recycling Conference, Wollongong, NSW, Australia, pp. 164-168, 2005. 
[5] National Research Council (NRC), Risk assessment in the Federal Government: managing the process, National Academy Press: Washington DC, 1983.

[6] Haas, C. N., Rose, J. B. \& Gerba, C. P., Quantitative Microbial Risk Assessment, John Wiley \& Sons Inc.: New York, 1999.

[7] Asano, T. \& Sakaji, R. H., Virus risk analysis in wastewater reclamation and reuse. Chemical, water and wastewater treatment, eds. H. H. Hahn \& R. Klute, Springer-Verlag: Berlin, Heidelberg. pp. 483-496, 1990.

[8] Asano, T., Leong, L. Y. C., Rigby, M. G. \& Sakaji, R. H., Evaluation of the California wastewater reclamation criteria using enteric virus monitoring data. Water Science and Technology, 26(7-8), pp. 1513-1524, 1992.

[9] Rose, J. B., Dickson, L. J., Farrah, S. R. \& Carnahan, R. P., Removal of pathogenic and indicator microorganisms by a full-scale water reclamation facility. Water Research, 30(11), pp. 2785-2797, 1996.

[10] Shuval, H. I., Lampert, Y. \& Fattal, B., Development of a risk assessment approach for evaluating wastewater recycling and reuse standards for agriculture. Water Science and Technology, 35(11-12), pp. 15-20, 1997.

[11] Tanaka, H., Asano, T., Schroeder, E. D. \& Tchobanoglous, G., Estimating the safety of wastewater reclamation and reuse using enteric virus monitoring data. Water Environment Research, 70(1), pp. 39-51, 1998.

[12] Van Ginneken, M. \& Oron, G., Risk assessment of consuming agricultural products irrigated with reclaimed wastewater: an exposure model. Water Resources Research, 36(9), pp. 2691-2699, 2000.

[13] Petterson, S. R., Ashbolt, N. \& Sharma, A. A., Microbial risks from wastewater irrigation of salad crops: A screening-level risk assessment. Water Environment Research, 72(6), pp. 667-672, 2001.

[14] Fegen, N., Gardner, T. \& Blackwell, P., Health risks associated with the reuse of effluent for irrigation - a literature review. State of Queensland Department of Natural Resources and Department of Primary Industries, 1998.

[15] Shuval, H. I., Yekutiel, P. \& Fattal, B., Epidemiological evidence for helminth and cholera transmission by vegetables with wastewater: Jerusalem - a case study. Water Science and Technology, 17, pp. 433442, 1984.

[16] Fattal, B., Wax, Y., Davies, M. \& Shuval, H. I., Health risks associated with wastewater irrigation: An epidemiological study. Am. J. Public Health, 76, pp. 977-979, 1986.

[17] Shuval, H. I., Investigation of typhoid fever and cholera transmission by raw wastewater irrigation in Santiago, Chile. Water Science and Technology, 27(3-4), pp. 164-174, 1993.

[18] Irving, L. G., Viruses in wastewater effluents (Chapter 7). Viruses and disinfection of water and wastewater, eds. M. Butler, A. R. Medlen \& R. Morris, University of Surrey, Guildford, 1982.

[19] Haas, C. N., Rose, J. B., Gerba, C. \& Regli, S., Risk assessment of virus in drinking water. Risk Analysis, 13, pp. 545-552, 1993. 
[20] Parkinson, A. \& Roddick, F. A., Wastewater treatment effectiveness: a review. Water, 31(5), pp. 63-68, 2004.

[21] Fattal, B., Goldberg, T. \& Dor, I., Model for measuring the effluent quality and the microbial die-off rate in Naan wastewater reservoir. Journal of Handasat Maim, 26, pp. 26-30, 1996.

[22] Schwartzbrod, L., Effect of human viruses on public health associated with the use of wastewater and sewage sludge in agriculture and aquaculture. World Health Organisation, Geneva. WHO/EOS/95.19, 1995.

[23] Petterson, S. R., Teunis, P. F. M. \& Ashbolt, N. J., B Modeling virus decay on salad crops using microbial count data. Risk Analysis, 21(6), pp. 1097-1108, 2001.

[24] US EPA, Guidelines for Reuse. EPA/625/R-92/004. Washington, DC. 247pp, 1992

[25] López, S., Prieto, M., Dijkstra, J., Dhanoa, M. S. \& France, J., Statistical evaluation of mathematical models for microbial growth. International Journal of Food Microbiology, 96, pp. 289-300, 2004.

[26] Rosebury, A. M. \& Burmaster, D. E., Log-normal distribution for water intake by children and adults. Risk Analysis, 21, pp. 99-104, 1992.

[27] Vose, D. J., The application of quantitative risk assessment to microbial food safety. Journal of Food Protection, 61(5), pp. 640-648, 1998.

[28] Furumoto, W. A. \& Mickey, R. A., Mathematical model for the infectivity-dilution curve of tobacco mosaic virus: experimental tests. Virology, 32, pp. 224-233, 1967.

[29] Ward, R. L., Bernstein, D. I., Young, E. C., Sherwood, J. R., Knowlton, D. R. \& Schiff, G. M., Human rotavirus studies in volunteers: determination of infectious dose and serological response to infection. Journal of Infectious Diseases, 154(5), pp. 871-880, 1986.

[30] Vose D., (2000) Risk analysis: A quantitative guide, John Wiley \& Sons Ltd: Chichester, 2000.

[31] Haas, C. N., Acceptable health risk. Viewpoint. Journal of the American Water Works Association, 18, pp. 8-10, 1996.

[32] WHO (2004a). Quantifying public health risk in the WHO guidelines for drinking-water quality: a burden of disease approach.

[33] WHO, Guidelines for drinking-water quality, 2004.

[34] Carr, R. M., Blumenthal, U. J. \& Mara, D. D., Health guidelines for the use of wastewater in agriculture: developing realistic guidelines, 2005. http://web.idrc.ca/es/ev-68330-201-1-DO TOPIC.html. 